\title{
LÉXICO DE LAS CAPITALES HISPANOAMERICANAS: PROPUESTA DE ZONAS DIALECTALES
}

\section{ANTECEDENTES}

Desde la muy conocida de Pedro Henríquez Ureña ${ }^{1}$, son múltiples las propuestas de zonas dialectales hispanoamericanas. Soy de la opinión de que casi todas aportan datos de interés para el mejor conocimiento del español americano, aunque también pueden verse en ellas errores evidentes, de forma y de fondo, de conformidad con lo que cada investigador entienda por zona dialectal. Si José Pedro Rona ${ }^{2}$ consideró defectuosa la división de Henríquez Ureña, especialmente por lo que concierne a su falta de sistematicidad, a su carácter no estructural, Juan Clemente Zamora y Jorge Guitart ${ }^{3}$ juzgaron asimismo que la de Rona tenía desaciertos graves, en particular su naturaleza no geográfica, habida cuenta de la no contigüidad de las áreas resultantes, así como también fue calificada como inconveniente la desproporción observable entre las superficies de las diversas zonas.

Por otra parte, es lógico que cada investigador, para fundar su propuesta, elija determinados rasgos lingüísticos diferenciadores. Algunos prefieren, como Rona, los aspectos estructurales (fonológicos o gramaticales, sobre todo), a otros, como a Henríquez Ureña, les parecen más útiles los fenómenos léxicos. Zamora y Guitart conceden igual importancia a características fonológicas que fonéticas o gramaticales. Aunque Henríquez Ureña

${ }^{1}$ En su artículo "Observaciones sobre el español en América", $R F E, 8$ (1921), 357-361.

2 "El problema de la división del español americano en zonas dialectales", en Presente y futuro de la lengua española (Actas de la Asamblea del I Congreso de Instituciones Hispánicas), Madrid, 1964, vol. 1, pp. 215-226.

${ }^{3}$ Dialectología Hispanoamericana, teoría, descripción, historia, Almer, Salamanca, 1982, pp. 179 ss. 
afirma que la base de su propuesta es el léxico ("el elemento di: tintivo entre dichas zonas está, sobre todo, en el vocabulario; e el aspecto fonético, ninguna zona me parece completamente un forme"'4, tengo la impresión de que se trataba más de una intu ción que del resultado de una verdadera comprobación empír $\mathrm{ca}^{5}$. En otras propuestas, carentes de datos obtenidos median encuestas dialectales, este carácter intuitivo es aún más evidentı

\section{2. ОвјЕto}

En las páginas que siguen pretendo ofrecer una propuesta m: de división dialectal del español americano. Si ésta tiene algur originalidad es la de basarse, por una parte, en el léxico culto e tándar de las capitales americanas y, por otra, que el corpus qu sirve para la división es producto de encuestas directas al esti de la dialectología tradicional.

Por lo que toca al primer aspecto, debe reconocerse que li hablas de las grandes ciudades tienen particular importancia $p$. ra la delimitación dialectal, si se acepta que se trata de dialectı normalmente muy influyentes. Suele verse en la norma de li grandes centros urbanos un crisol donde, por una parte, se fu den las hablas de los muchos dialectos a los que pertenecen li numerosos inmigrantes que continuamente arriban a las pobl ciones que ofrecen mayores posibilidades de empleo o de subsi tencia; pero, por otra parte, estas concentraciones urbanas sc asimismo focos irradiadores de influencia lingüística en zonas c ya extensión está con frecuencia en relación directamente pr porcional con la importancia de la ciudad de que se trate. Es p tanto probable que tenga cierto interés el estudio comparado $d$ léxico de las ciudades capitales para observar si es posible agr parlas de forma tal que constituyan núcleos de zonas dialectal cuyas isoglosas estarían dadas por semejanzas y diferencias c vocabulario. Parto para ello de la hipótesis de que el léxico está dar de una determinada capital americana está necesariamen más relacionado o, si se quiere, tiene más semejanza con el léxi de tal o cual capital que con el vocabulario del resto de las ciud

${ }^{4}$ Art. cit., p. 358.

${ }^{5}$ Puede verse, por una parte, que el propio investigador juzgaba pro sional su propuesta y, por otra, llama la atención la falta de ejemplos léxic que apoyen su propuesta de división dialectal. 
des. Nada impide formular la existencia de áreas dialectales que tengan como núcleo o núcleos una o varias poblaciones importantes y cuyas isoglosas queden determinadas por diferencias léxicas. Véase un simple ejemplo: parece lógico vincular, por semejanzas en su vocabulario, ciudades como Santo Domingo, San Juan y La Habana, mejor que formar un grupo constituido por La Habana, Lima y Montevideo. Sin embargo, por una parte, esta intuición debe demostrarse y, por otra, resultaría ya más difícil de acertar, por mera intuición, si se pregunta cuál dialecto, en cuanto a su vocabulario, es más semejante al de Santo Domingo, si el de La Habana o si el de San Juan. Pretendo en este estudio, primero, ver si en efecto existe, para seguir con el ejemplo, una semejanza tal entre esos tres vocabularios (el de Santo Domingo, San Juan y La Habana) que permita la formulación de una zona dialectal antillana; y, segundo, observar las relaciones de semejanzas y diferencias internas en la zona, es decir entre los léxicos de las tres ciudades entre sí. Este tipo de confrontaciones abarcarán la totalidad de las capitales americanas.

Por lo que respecta al tipo de léxico que se estudiará, conviene destacar que se trata de un vocabulario estándar. Entiendo por ello, conceptos y designaciones que pertenecen a la mayoría de los idiolectos que constituyen los dialectos urbanos. Juzgo que esta característica resulta indispensable si se desea comparar debidamente los léxicos de las ciudades capitales. En otras palabras, los conceptos o referentes que se tomarán en consideración son comunes a la mayor parte de los hablantes de todas las ciudades investigadas y tienen por ende una enorme difusión en cada uno de los dialectos. Se trata de conceptos comunes y corrientes, o de $r a$ rismos propios de cierta región o de determinado grupo de hablantes. Esta característica permite, por una parte, que la totalidad de los sujetos esté en capacidad de contestar las preguntas, lo que quiere decir que las voces recogidas pertenecen de hecho a cada una de las normas urbanas estudiadas; y, por otra, que pueda hacerse una efectiva y válida comparación entre los vocabularios de las diversas normas.

\section{MÉTODO}

Se aplicó una parte del Cuestionario léxico que se emplea para el "Proyecto de estudio coordinado de la norma lingüística culta de las principales ciudades de Iberoamérica y la Península Ibéri- 
ca"6. Primeramente, del total de 4,452 cuestiones, se hizo ur selección de sólo 292. El motivo, para esta primera reducciór fue simplemente elegir sólo aquellas cuestiones que, por una pa te, no presentaran particular dificultad para ningún sujeto $c$ cultura media ${ }^{7}$; y solamente las que, por otra, ofrecieran cier garantía de variación en la geografía americana ${ }^{8}$.

Hubo necesidad, después, de eliminar algunas otras pregm tas. Esta segunda reducción tuvo que ver con el hecho de q1 ciertas respuestas tenían alguna de las siguientes característica a pesar de haber operado ya la primera selección, las respuestas algunas cuestiones fueron semejantes en todos los sujetos encue tados, aunque con frecuencia se trataba de formas léxicas dif rentes de las que prevalecen en la Península Ibérica, en Madri particularmente. Eran por tanto conceptos que permitían form dos bloques: España y América ${ }^{9}$. Si la intención era encontr. zonas dialectales americanas, es lógico que se haya suprimido e te tipo de cuestiones. Finalmente, eliminé de la lista algunos $\mathrm{p}$ eos conceptos que ciertamente dieron lugar a vocablos diferente sin embargo, cuando traté de agruparlos, observé que aparecía dispersos en la amplia geografía americana pero que con ellos I podía constituirse ni siquiera una sola zona. En otras palabra las ciudades donde se registraba una misma respuesta no teníc contigüidad.

${ }^{6}$ Noticia completa de este proyecto puede leerse en JuAn M. LC BLANCH, El estudio del esapñol hablado culto, historia de un prayecto, UNAM, N. xico, 1986.

${ }^{7} \mathrm{Se}$ evitaron por tanto todas las preguntas que de una $\mathrm{u}$ otra forma $\mathrm{pt}$ den considerarse especializadas, como podrían serlo muchas de las referente: asuntos financieros, por ejemplo.

${ }^{8}$ Esto quiere decir que se omitieron todas las preguntas que, así sea $\mathrm{f}$ mera intuición, se supone que se manifiestan por una misma forma léxica cualquier parte, como por ejemplo EL ESQUELETO, EL CRÁNEO, H TEL, LA FRUTA, EL VINO, PANTALÓN, LA GAMISA, EL A QUITECTO, EL ALBAÑIL, etcétera.

${ }^{9}$ Casi nunca se trata de conceptos que recibieran la misma respuesta parte de todos lo sujetos investigados; sin embargo puede asegurarse que exi una voz predominante en Madrid y otra, igualmente predominante, en An rica. Algunos ejemplos (en adelante escribiré con mayúsculas los concepto con cursivas los vocablos): MEJILLAS (mejillas, en Madrid; cachetes, en Ar rica); JUGO (lo que se obtiene si se exprime una naranja) (zumo frente a juछ ORFANATO (orfanato, orfelinato, contra orfanatorio); AUTOMÓVIL DI CAPOTABLE (descapotable; convertible); RADIOS DE LA BICICLETA । dios; rayos); ALCANCÍA (receptáculo cerrado con una abertura estrecha ] donde se echa dinero para guardarlo) (hucha; alcancía)... 
Aclaro de inmediato que para fines de este estudio entiendo por ciudades capitales contiguas todas aquellas entre las cuales no se interpone ninguna otra ciudad capital; que forman, por así decirlo, una cadena. Esto quiere decir que si, para un concepto A hubo una respuesta predominante $\mathrm{B}^{10}$ en México y Guatemala, otra respuesta C en San Salvador, y otra respuesta D en Managua, Tegucigalpa, San José y Panamá, se considerarán relacionadas sólo, por una parte, las ciudades de México y Guatemala y, por otra, Managua, Tegucigalpa, San José y Panamá. Mientras se cumpla este principio de contigüidad, podrán formarse cadenas de ciudades enlazadas o relacionadas de diversa extensión, de dos ciudades en adelante, sin que la cadena pueda abarcar nunca todas las capitales porque, como ya dije, las cuestiones que obtuvieron respuestas semejantes en todo el continente fueron suprimidas de la lista. Hubo por tanto algunos conceptos que obtuvieron respuestas diferentes pero que en ningún caso establecieron cadenas de al menos dos ciudades; fueron por ello suprimidos ${ }^{11}$.

Como se comprenderá, este principio de contigüidad es de particular interés, en este estudio, para el establecimiento de zonas dialectales. Hay casos en los cuales la aplicación del principio de contigüidad no parece presentar dificultad alguna. Claramente contiguas parecen serlo, entre sí, las capitales centroamericanas, las ciudades capitales de las islas antillanas (La Habana, Santo Domingo y San Juan), las capitales de la región del Plata (Montevideo y Buenos Aires), etcétera. No puede decirse esto de otros agrupamientos. Como simples ejemplos, puesto que cada

${ }^{10}$ Aclaro de paso que el concepto de respuesta predominante tiene interés en este estudio. Con ello quiero significar que, cuando se registran, para un mismo concepto, varias respuestas en un solo punto de encuesta, la única voz que se considera para fines de comparación, o de formación de áreas dialectales será la predominante, que necesariamente debe ser superior al cincuenta por ciento de las respuestas obtenidas.

11 Véase algún ejemplo: aunque para el concepto MELOCOTÓN, además de durazno, que fue la designación americana más general, casi continental (y que por ello no da lugar a zonas dialectales), se documentó también la voz melocotón, ello sucedió sólo en San Salvador, San Juan y Lima, que no pueden considerarse contiguas, y por tanto se decidió suprimir ese concepto de la lista. EL ÚLTIMO PISO DE UN EDIFICIO DE VIVIENDAS se designa, continentalmente, por el anglicismo penthouse. También se documentó la voz último piso, en México y San Salvador (no contiguas) y terraza, en Managua, La Paz, Asunción y Buenos Aires (tampoco contiguas); se eliminó de la lista este concepto. 
caso se discutirá después particularmente, véase que Méxicr puede considerarse, de conformidad con el criterio establecido contiguo tanto de las capitales centroamericanas cuanto de la antillanas ${ }^{12}$; existe asimismo la duda de si Caracas se relacion: más con Bogotá o con las capitales de las Antillas, especialmentı San Juan; puede uno preguntarse si Santiago, en cuanto al léxic considerado, está más próximo a Lima, a La Paz, a Asunción . a Buenos Aires, etcétera. Como se ve, la dificultad no está en es tablecer contigüidades geográficas, que ya están claramente esta blecidas, sino en la manera como puedan vincularse, por seme janzas léxicas, dos, tres o más ciudades americanas.

$\mathrm{Si}$ se considera además que fueron suprimidos los concepto que produjeron respuestas continentales, se comprenderá la dificul tad de establecer límites más o menos precisos a la dimensión d las zonas dialectales que se lleguen a proponer: ¿dos ciudade constituyen una zona? ¿todas las capitales sudamericanas dan lu gar a otra? Es obvio que las respuestas a estas preguntas consti tuirán propiamente el cuerpo del estudio.

En definitiva, habida cuenta de las diversas supresiones, $\epsilon$ total de conceptos con los que se trabajará es sólo de $232^{13}$. S hicieron al menos dos encuestas por cada ciudad capital ${ }^{14}$. El lé xico de algunas capitales resultó mejor conocido por haberse he cho ahí mayor número de encuestas. Tal es el caso, sobre todc

12 Es decir que, entre México y las capitales antillanas se interpone cie: tamente el mar, pero ninguna otra ciudad capital; asimismo puede relaciona: se la capital mexicana con Guatemala (y con las demás capitales de Gentro: mérica, hasta que exista una interrupción). El problema estará en decidir s de conformidad con los resultados de las encuestas, el léxico mexicano se parı ce más al antillano o al centroamericano, con el fin de decidir si hay o no r: zón para proponer una zona dialectal en la que quede comprendido Méxic y las Antillas, o México y Centroamérica, o México, las Antillas y Centro: mérica, o si simplemente, no hay razones para tal proposición y México : deba considerar no propiamente aislado, sino más bien constituyendo en mismo una sola zona dialectal.

13 Agrupables en los siguientes campos semánticos: el cuerpo human la alimentación, el vestuario, la casa, la familia, el ciclo de la vida, la salur la vida social, diversiones, la ciudad, el comercio, transportes y viajes, los $\mathrm{m}$ dios de comunicación, comercio exterior, política nacional, sindicatos y cor perativas, profesiones y oficios, mundo financiero, la enseñanza, la iglesii meteorología, el tiempo cronológico, el terreno, vegetales, agricultura, an males, ganadería.

${ }^{14}$ Con sujetos oriundos de cada ciudad capital y que, en el momento c la encuesta, no tenían más de dos años de vivir en la ciudad de México, done se llevaron a cabo la totalidad de los interrogatorios. 
de México y de Santiago, ciudades de cuyo léxico se han publicado sendos libros, que contienen las respuestas ordenadas de todo el cuestionario, aplicado a un buen número de sujetos ${ }^{15}$. Obtuve respuestas para todas y cada una de las capitales americanas ${ }^{16}$.

\section{MÉxico}

Hubo un total de 149 casos de coincidencia de respuestas mexicanas con las de otro país contiguo, entendiendo por ello, en este caso particular, cualquier capital centroamericana o del área antillana. De este total, se registraron 50 (es decir un 33.5\%) en que la coincidencia fue solamente con capitales centroamericanas. Sólo 8 casos hubo de coincidencia exclusiva con capitales de las islas antillanas (5.3\%). En 61 casos $(40.9 \%)$ se documentaron coincidencias con capitales centroamericanas y simultáneamente con capitales de otros países (sudamericanos o antillanos). Finalmente, en 30 ocasiones $(20.1 \%$ ) las coincidencias fueron, por una parte, con capitales antillanas y, simultáneamente, con capitales centro o sudamericanas. Es evidente por tanto que hay mayor semejanza entre el léxico de México y el de Centroamérica que entre el de aquella ciudad y el de las capitales antillanas. Por otra parte, probablemente la ciudad de México pueda verse, en esa zona, como el foco difusor de más importancia, si se considera, primero, que es el habla que establece mayor número de coincidencias con el vocabulario de otras ciudades capitales "vecinas" (centroamericanas y antillanas) y, segundo, que también se trata de la ciudad que registra un más alto número de respuestas "exclusivas", no documentadas en otras capitales americanas ${ }^{17}$, o quizá, por esta última razón, convendría considerar a

${ }^{15}$ Las fichas bibliográficas correspondientes son: JUAN M. LOPE B BANCH (ed.), Léxico del habla culta de México, UNAM, México, 1978. Ambrosio Rabanales y Lidia Contreras, Léxico del habla culta de Santiago de Chile, UNAM, México, 1987.

${ }^{16}$ Se entiende que me refiero a capitales americanas hispanohablantes, que trataré de citar siempre en orden descendente de norte a sur, comenzando por México y terminando en Buenos Aires.

17 Debe entenderse que lo de exclusivo se reduce, obviamente, a los límites de las encuestas aplicadas. Es muy probable que, de haberse aplicado el cuestionario a un mayor número de sujetos de las diversas capitales, no pocas de las respuestas que aquí denomino "exclusivas" dejarían de serlo. Sin embargo es importante asimismo destacar que esta misma condición impera para todas las demás capitales, por ende los resultados no dejan, a mi ver, de tener 
esta ciudad, por sí sola, como una zona dialectal completa, sepa rada de las demás.

De las 292 preguntas de que constaba el cuestionario comple to, hubo 44 (14.9\%) que tuvieron en México respuestas no docu mentadas en ninguna otra capital. Considérese, por ejemplo que de ese mismo total, Guatemala sólo obtuvo 16 respuesta "exclusivas" (5.4\%). Antes de seguir refiriéndome a las rela ciones entre el léxico de la ciudad de México y el propio de Cen troamérica y las Antillas, proporcionaré algunos ejemplos d conceptos que tuvieron, en México, una respuesta que podrí considerarse exclusiva: LIMPIAR LOS ZAPATOS CON CRE MA, en México: bolear ${ }^{18}$; SELLO DE CORREO: timbre $^{19}$; El PELO DE LOS NEGROS: chino ${ }^{20}$; VUELTA O DOBLADI LLO DEL PANTALÓN: valenciana ${ }^{21}$; AUTOBÚS URBANO camión $^{22}$; EL GUARDABARRO: salpicadera ${ }^{23}$; PELO RUBIO güero $^{24}$; PERSONA A LA QUE LE FALTA UN DIENTE: chi muelo $^{25}$; EL LIMPIABOTAS: bolero $^{26}$; MALETERO (comparti miento de los autos para maletas o paquetes): cajuela ${ }^{27}$; BOLj

un buen grado de confiabilidad.

${ }^{18}$ Lustrar fue la respuesta predominante en las capitales americana: chainear (Tegucigalpa), brillar (San Juan), pulir (Caracas), embolar (Bogotá). .

${ }^{19}$ Estampilla es la designación más frecuente en América; sello (Guatem: la, La Habana, Santo Domingo, Montevideo)...

${ }^{20}$ Crespo (San José, Botogá, Lima, La Paz), pasa (La Habana, Santo Dr mingo), zambo (Quito, Lima)...

${ }^{21}$ Ruedo (Guatemala, San Salvador, Tegucigalpa, Managua, San Josi Santo Domingo, San Juan, Asunción), basta (Panamá, Quito, Lima, Santic go), botamanga (Asunción, Buenos Aires)...

${ }^{22}$ Camioneta (Guatemala, San Salvador), bus (Tegucigalpa, Managu: Panamá, La Habana, Bogotá, Quito), guagua (La Habana, Santo Domingc San Juan), colectivo (La Paz, Asunción, Buenos Aires), micro (Santiago).

${ }_{23}$ Guardafango (San Salvador, Tegucigalpa, Managua, San José, Pani má, La Habana, Santo Domingo, San Juan, Caracas, Quito, Lima, La Paz guardabarro (Bogotá, Asunción, Montevideo, Buenos Aires)...

${ }^{24}$ Rubio (Managua, La Habana, Santo Domingo, San Juan, Quitı Santiago, Buenos Aires), canche (Guatemala), chele (San Salvador, Teguciga pa), fulo (Panamá), choco (La Paz)...

${ }_{25}$ Mellado (San Juan), choleo (Guatemala, San Salvador), bichino (Teguc galpa), chintano (Managua), mueleta (San José), bocacho (Panamá), desdenta (La Habana, Santo Domingo, Lima, Asunción, Montevideo), mueco (Bogr tá), casadiente (La Paz)...

${ }^{26}$ Limpiabotas (en muchas capitales americanas), lustrador (Guatemal: San Salvador, Managua), lustrabotas (Tegucigalpa, La Paz, Santiago, Asuı ción, Montevideo, Buenos Aires), embolador (Bogotá), canilleta (Lima)...

${ }^{27}$ Maletero o maletera (La Paz, Panamá), baúl (Guatemala, San Salvado 
GRAFO: pluma atómica28; MARIQUITA (insecto rojo con puntitos negros): catarina $^{29}$; ORZUELO (grano que a veces sale en el borde de las párpados): perrilla ${ }^{30}$; LAS AMÍGDALAS: anginas $^{31}$; CORDONES DE LOS ZAPATOS: agujetas $^{32}$; PISCINA: alberca ${ }^{33}$; TIZA: gis ${ }^{34}$; MICO, MONO: chango ${ }^{35}$.

Por lo que respecta a las relaciones del léxico de la capital mexicana con el propio de las capitales centroamericanas, debe anotarse que en algunos pocos conceptos hubo semejanza entre México y las capitales de los seis países del área, hubo asimismo casos en que la coincidencia se da sólo con cinco, cuatro, tres, dos o una sola de las ciudades capitales. Si se analiza la relación de ciudad a ciudad, el orden descedente de coincidencia es el siguiente: con Managua (51 casos), Guatemala (47), San Salvador (46), Tegucigalpa (37), San José (32) y Panamá (30).

Transcribo a continuación algunos ejemplos de conceptos que recibieron respuestas exclusivas de México y algunos países centroamericanos, no documentadas en las encuestas de otras ciudades americanas: LOBANILLO (protuberancia sebácea superficial que se forma en algunas partes del cuerpo): mezquino (en México, Guatemala, San Salvador, Tegucigalpa, Managua, San José) ${ }^{36}$; INDIVIDUO PEQUEÑO: chaparro (México, Guate-

Tegucigalpa, La Habana, Santo Domingo, San Juan, Quito, Buenos Aires), valijera (Managua, Asunción)...

${ }^{28}$ Bolígrafo (en muchas capitales americanas), lapicero (Guatemala, Managua, La Habana, Lima), esferógrafo o esferográfica (Bogotá, Quito), puntabola (La Paz), lápiz tinta (Tegucigalpa), lápiz de pasta (Santiago), birome (Asunción, Buenos Aires)...

${ }^{29}$ Mariquita (en varias capitales), tortolita (Guatemala, San José), tortuguita (Tegucigalpa, Managua), vaquita (San José), cucarrón (Bogotá), coroncho (Caracas), lorito (La Paz), chinita (Santiago), sanantonio (Montevideo), juanita (Buenos Aires).

${ }^{30}$ En casi todas partes: orzuelo; escupelo (Guatemala), chalazo (La Habana)...

31 Amigdalas es la respuesta de todas las otras capitales; glándulas, en Asunción.

32 Cordones (frecuente en muchas ciudades), correas (Guatemala), gavetes (San Juan), trenzas (Caracas), pasadores (Lima), guatos (La Paz).

${ }^{33}$ En casi todas partes: piscina; pileta (Asunción, Montevideo, Buenos Aires)...

${ }^{34}$ En casi todas partes predomina el nahuatlismo tiza; yeso (Guatemala, San Salvador, Tegucigalpa).

${ }^{35}$ La designación común es mono; mico (Guatemala, Managua, Bogotá).

${ }^{36}$ Verruga en Quito, Lima, La Paz, Montevideo, Buenos Aires; ojo de pescado en La Habana y Santo Domingo. 
mala, Tegucigalpa, Managua) ${ }^{37}$; EL INTERRUPTOR DE L $t$ LUZ: apagador (México, San José y Panamá) 38 ; EL CAROZO CORONTA, TUSA DE LA MAZORCA (el soporte de los gra nos): olote (México, San Salvador, Tegucigalpa, Managua, Sa José) ${ }^{39}$; MAZORCA TIERNA: elote (México, Guatemala, Sa Salvador, Tegucigalpa, Managua, San José) ${ }^{40}$; MORCILL $t$ (embutido de sangre con aliños): moronga (México, Guatemala San Salvador, Tegucigalpa, Managua ${ }^{41}$; BOLSILLO DE] PANTALÓN: bolsa (México, Guatemala, San Salvador, Teguci galpa, Managua, San José) ${ }^{42}$; EL CALENTADOR DE AGUA boiler (México, Managua, San José) ${ }^{43}$; LA RADIO: el rade (México, Tegucigalpa, Managua, San José) ${ }^{44}$.

Algunos ejemplos de coincidencias, más raras, entre Méxic y las capitales antillanas, en voces no registradas en otras ciuda des: GUISANTES, CHÍCHAROS, ARVEJAS: chicharos (Mé xico, La Habana) 45 $^{45}$ BRAGAS (de mujer): pantaletas (Méxicc Santo Domingo) ${ }^{46}$; LA CERA (secreción de los oídos): cerill (México, La Habana, Santo Domingo, San Juan) ${ }^{47}$.

${ }^{37}$ Bajo, chico, enano, en muchas partes; topito, en San Juan; chato, en L ma y La Paz; petiso, en La Paz, Asunción, Montevideo y Buenos Aires.

${ }^{38}$ Interruptor (La Habana, Caracas, Quito, La Paz, Santiago); swite (Santo Domingo, San Juan, Bogotá); llave de la luz (Lima, Asunción, Monte video, Buenos Aires).

${ }^{39}$ Carozo (Buenos Aires); tusa (La Habana, San Juan, Caracas, Quito coronta (Santiago); marlo (Asunción, Montevideo).

${ }^{40}$ Mazorca (tierna) (Santo Domingo, Bogotá); maíz tierno (La Habana San Juan); choclo (Quito, Lima, La Paz, Santiago, Asunción, Montevider Buenos Aires); jojoto (Caracas).

${ }^{41}$ Morcilla (en muchos lugares); pichoón (Caracas); relleno, moshinga (L ma); prieta (Santiago). Aires)

${ }_{42}$ Bolsillo (en la mayor parte de las capitales); cartera (Santiago, Buenc

${ }^{43}$ Calentador (en muchas partes); terma (Lima); calefón (La Paz, Santiagc Buenos Aires); termocalefón (Asunción); termotanque (Buenos Aires).

${ }^{44}$ En las demás partes o predomina la radio o alternan el radio y la radie

${ }^{45}$ Arvejas (predominante en las capitales americanas); petitpois (Manz gua, Panamá).

${ }^{46}$ Calzón (es) (Guatemala, Managua, San José, Lima, La Paz, Santic go); blúmer (San Salvador); short(s) (Tegucigalpa, Bogotá); panti(es) (Panamá Caracas); medias (Quito); cuadros (Santiago); bombacha(s) (Asunción, Montev deo, Buenos Aires).

${ }^{47}$ Cera (predominante en la mayoría de las ciudades americanas); cer men (Guatemala); cerra (San José, Montevideo). 


\section{Centroamérica}

No parece aventurado proponer, como zona dialectal, la región centroamericana, si se considera que el léxico de las capitales de esos países tiene entre sí evidentes afinidades. Esto quizá pueda quedar probado por el simple hecho de que cada una de estas ciudades establece, con las demás ciudades del área, un mayor número de relaciones que con cualquier otra capital americana. El argumento podría expresarse en los siguientes términos: para siete conceptos del cuestionario las seis capitales de Centroamérica presentaron una misma respuesta predominante; hubo 16 casos en que la misma respuesta pertenece a cinco de las seis capitales; 30 preguntas tuvieron respuestas análogas en cuatro ciudades; en 52 cuestiones se dio la misma respuesta en tres de esas capitales; finalmente, hubo 93 casos en que la asociación se limita a sólo dos capitales. El número de asociaciones que pudieran formarse entre, por ejemplo, Guatemala y cualquier otra capital que no sea centroamericana será siempre muy inferior al que se obtiene cuando se consideran las afinidades del léxico de esa capital con el propio de las demás capitales del área. Lo mismo puede decirse de las demás ciudades ${ }^{48}$.

Podría resultar de interés observar la manera como se relacionan o se agrupan entre sí las diversas capitales de esta región. Si se separan los conceptos que recibieron la misma respuesta en cinco de las seis ciudades, la agrupación más frecuente $(50 \%$ aproximadamente) es la que elimina a la ciudad de Panamá, lo que parece explicable en cuanto que parece tratarse de un léxico, el panameño, en cierta forma fronterizo entre centro y Sudamérica. Por lo que toca a los casos en que hubo semejanzas en cuatro de las seis capitales, el grupo más frecuente (30\%) es el constituido por Guatemala, San Salvador, Tegucigalpa y Managua, seguido muy de cerca (27\%) por el que forman San Salvador, Tegucigalpa, Managua y San José. Si se consideran agrupamientos de sólo tres capitales, hay evidente predominio $(21 \%)$ del que forman Guatemala, San Salvador y Tegucigalpa ${ }^{49}$. Finalmente,

${ }^{48}$ En efecto, hay casos como el siguiente: para el concepto VITRINA O ESCAPARATE se obtuvo en Managua la respuesta aparador, también registrada en México y en Lima, y no documentada en otras capitales centroamericanas. Estos casos, sin embargo, deben considerarse excepcionales.

${ }^{49}$ Podría pensarse que es un porcentaje bajo el $21 \%$. Téngase en cuenta empero que son muchas las ternas que se pueden formar con seis capitales. Muchas de esas ternas o no se dieron (por ejemplo la de Guatemala, San José 
las más frecuentes combinaciones de dos capitales centroamer canas, con el mismo porcentaje ambas (15\%) son las que se dar por una parte, entre Guatemala y San Salvador y, por otra, entr San Salvador y Tegucigalpa ${ }^{50}$.

Véanse algunos ejemplos de conceptos, cuyas respuestas, $\dot{c}$ conformidad con los resultados de mis encuestas, parecen excl sivas de algunas capitales centroamericanas. TAPAS (cosas qu se sirven en un aperitivo, fuera de los licores): bocas o boquiti (Guatemala, San Salvador, Tegucigalpa, Managua, San J sé) ${ }^{51}$; AUTOBÚS: camioneta (Guatemala, San Salvador) ${ }^{52}$; Pf VO: chompipe (Guatemala, San Salvador, Tegucigalpa, Man: gua, San José) $)^{53}$; LUGAR PARA TOMAR BEBIDAS D INFIMA CALIDAD: experidio estanco (San Salvador, Teguciga pa) ${ }^{54}$; EL LAVADERO, LA PILETA: pila (Guatemala, teguc galpa, San José) ${ }^{55}$; LUZ INTERMITENTE (la que, en el aut móvil, se prende y se apaga continuamente para cambiar c dirección): pide vías (Guatemala, Managua) ${ }^{56}$; SUJETACOI BATA: prensacorbata (Guatemala, Tegucigalpa, Managua) ${ }^{5}$ APUNTACIONES FRAUDULENTAS PARA EL EXAMEI

y Panamá) o aparecieron sólo una vez (como sería el caso de Guatemala, M nagua y Panamá).

${ }^{50}$ Probablemente la combinación menos frecuente entre dos capita sea la de Panamá y San Salvador, registrada sólo una vez.

51 Botanas (México), saladitos (La Habana), bocadillos o bocaditos (Sar Domingo, Lima, La Paz, Asunción), entremeses (San Juan, Quito, La P: Santiago, Asunción), picadas (Bogotá, Montevideo), pasapalos (Caracas), pa bocas (Bogotá), etcétera.

${ }^{52}$ Autobús (en varios sitios); camión (México); guagua (La Habana, Sar Domingo, San Juan); ómnibus (Lima, Asunción, Montevideo, Buenos Aire Aunque también documentada en Bogotá, Quito y Santiago, la respuesta se dio en varias capitales centroamericanas: San Salvador, Tegucigalpa, lv nagua, San José y Panamá.

${ }^{53}$ Pavo es la respuesta más común en otras ciudades; guajolote, cócono, pilo, totol (México); guanjo (La Habana).

${ }^{54}$ Cantina (la respuesta más frecuente); cafetín (San Juan); tugurio, tagu (Caracas); chichería (Quito, La Paz); picantería (Lima).

${ }_{55}$ Esta misma voz (pila) es usual en Madrid. Otras respuestas ameri nas: lavadero (la más común); pileta (La Habana, Asunción, Montevideo, B nos Aires); batea (Caracas); piedra de lavar (Quito).

${ }^{56}$ Intermitentes (la designación más frecuente); direccionales (México, Sa: Domingo, Lima); signal lights (San Juan); guiñadores (La Paz); señalero (Ası ción, Montevideo); balizas (Buenos Aires)...

${ }^{57}$ Pisacorbata (México, Santo Domingo, Bogotá, Caracas); pasador ( Habana, Santiago); pinche de corbata (San Juan); prendedor (Quito); alfiler (M. tevideo); aprietacorbata (Asunción); traba (Buenos Aires, Montevideo). 
copias (San Salvador, Managua) ${ }^{58}$; PANTALÓN DE BAÑO: calzoneta (Guatemala, San Salvador, Tegucigalpa, Managua) ${ }^{59}$; LAS CANICAS: chibolas (San Salvador y Managua) ${ }^{60}$.

\section{Las Antillas}

No cabe duda de que las tres capitales de las Antillas Mayores (La Habana, Santo Domingo y San Juan) constituyen, de conformidad con el corpus léxico que estoy considerando, una zona dialectal. En combinaciones de tres ciudades, cada una de estas capitales entran en relación con las otras dos con evidente mayor frecuencia que con otras ciudades capitales americanas; lo mismo sucede con los numerosos casos en que un concepto recibe una misma respuesta en más de tres ciudades: es normal que en tales casos aparezcan juntas las tres capitales antillanas. Algo análogo sucede si se consideran sólo parejas de ciudades: Santo Domingo, sea por caso, se relaciona mayormente con La Habana y con San Juan más que con cualquiera otra capital del continente.

El caso de la ciudad de Caracas es más difícil. Según mis resultados, el léxico estándar de la capital venezolana parece asemejarse más al del área de las islas antillanas que al propio de otras zonas sudamericanas. Podría uno preguntarse, por ejemplo, si hay más semejanzas léxicas entre Bogotá y Caracas o entre esta ciudad y San Juan. Soy de la opinión de que sucede lo segundo, aunque se necesitaría un mayor volumen de datos dialectales para afirmarlo con cierta seguridad. Tentativamente, por tanto, propongo integrar en una sola zona dialectal las tres capitales de las islas antillanas y la capital de Venezuela.

Ahora bien, por lo que respecta a las relaciones internas que pueden observarse en la zona, hay diferencias obvias en cuanto al grado de cercanía (geográfica y lingüística) entre unas ciuda-

${ }^{58}$ Acordeones (México, San Juan, Buenos Aires); fraude (La Habana, Bogotá); chivos (Santo Domingo); drogas (San Juan); pollas (Quito), comprimidos (Lima); chanchullos (La Paz); torpedos (Santiago); copiatini (Asunción); ferrocarril (Montevideo); machetes (Buenos Aires).

59 Traje de baño (frecuente en varias partes); vestido de baño (Bogotá); malla (La Paz, Asunción, Buenos Aires).

${ }^{60}$ Canicas (México, Santo Domingo, San Juan, Lima, La Paz); bolas (La Habana, Quito, Lima, La Paz, Santiago, Asunción, Montevideo, Buenos Aires); metras (Caracas)... 
des y otras. Limitándome a las tres capitales antillanas, excluye do por el momento a Caracas, debo señalar que la relación m: fuerte se establece entre Santo Domingo y San Juan (en un 49.1 de los casos), seguida de la que se da entre La Habana y San Domingo (30\%), siendo la más débil (apenas un 20.3\%) la qi se produce entre La Habana y San Juan. Finalmente, en que toca a las semejanzas léxicas entre Caracas y las ciudades a] tillanas, éstas se muestran más o menos evidentes entre Carac: y San Juan; y escasas entre la capital venezolana y la dominicar y, sobre todo, la cubana, con cuyo léxico el vocabulario caraqu ño (dialectal, se entiende) tiene realmente pocas afinidades.

Transcribo en seguida algunos ejemplos de conceptos pa: los cuales se obtuvieron respuestas al parecer característic (aunque quizá no necesariamente exclusivas) de esta zona diale tal antillana, con la ciudad de Caracas incluida: EL SITIO Ps RA CONSTRUIR (trozo de tierra sin construcción): solar (Sa: to Domingo y San Juan) ${ }^{61}$; EL EMBRAGUE (DEL AUTC CLUTCH: cloche (Santo Domingo, San Juan, Caracas) ${ }^{62} ; \mathrm{S}$ LLÍN (DE LA BICICLETA): sillin (Santo Domingo, Si Juan) ${ }^{63}$; EL PELO DE LOS NEGROS: pasa (La Habana, Sa to Dominto) ${ }^{64}$; AUTOBÚS URBANO: guagua (La Haban Santo Domingo, San Juan $)^{65}$; CALLE DE DOBLE VÍA DOS DIRECGIONES: de dos vías (La Habana, Santo Domi go $)^{66}$; PATIO DE BUTACAS (planta baja del teatro): primer 1 so (Santo Domingo, Caracas) ${ }^{67}$; GEMELOS (para los puños ، la camisa): yuntas (San Juan, Caracas) ${ }^{68}$; BOMBILLA: bombi

${ }^{61} \mathrm{Voz}$ también usual en Madrid. Otras respuestas: terreno (en varias c: dades), lote (Tegucigalpa, San José, Bogotá), predio (Buenos Aires), bal (Asunción).

${ }^{62}$ Embrague (Quito, Lima, La Paz, Santiago, Asunción, Montevid Buenos Aires); clutch (pronunciado a la inglesa), en otras varias capitales.

63 Asiento (predominante en América); sillón (Guatemala, Panamá).

llín, como segunda opción, apareció en varias otras ciudades.

64 Véase la n. 20.

65 Véase la n. 22.

${ }^{66}$ De doble sentido (México, La Paz, Asunción); de doble vía (varias ciuc des), de doble dirección (Montevideo); de doble mano (Buenos Aires).

67 Luneta (México, Guatemala, San Salvador, Tegucigalpa, Manag San José, Panamá, Quito); palcos (Guatemala, Montevideo); platea (Bogo La Paz, Asunción); primera fila (Panamá, Montevideo)... .

${ }^{68}$ Gemelos (Santo Domingo, Lima, Montevideo, Asunción, Buenos 4 res); mancuernillas (México, Guatemala, Tegucigalpa, San Salvador, Maı gua, San José); broches (Quito); clips (La Paz); colleras (Santiago). 
(Santo Domingo, Caracas) ${ }^{69}$; ALMACÉN DE VÍVERES: colmado (Santo Domingo, San Juan) ${ }^{70}$; EL VOLANTE (del automóvil): guía (Santo Domingo, San Juan) ${ }^{71}$; MAZORCA TIERNA: maiz tierno (La Habana, San Juan) ${ }^{72}$; EL TRAJE (conjunto de piezas que forman el ropaje externo del hombre): flux (Santo Domingo, San Juan, Caracas) ${ }^{73}$.

\section{Bogotá, Quito, Lima y la Paz}

Es probable que las capitales del extenso territorio de Sudamérica puedan agruparse de muy diversas formas. Presento sin embargo la que, según mis materiales léxicos, parece más coherente. Habiendo decidido dejar a Caracas como parte de la zona antillana, el resto de las capitales de América del Sur quedarán agrupadas en dos conjuntos. En el primero incluyo a Bogotá, Quito, Lima y La Paz; en el segundo queda el resto de las capitales, ubicadas en la parte meridional del continente, conocida también como Cono Sur.

De las ciudades que forman esta primera zona, juzgo que es Bogotá la de más difícil inclusión. Sin embargo prefiero dejarla aquí y no en otro sitio: no se relaciona satisfactoriamente ni con Caracas (y las Antillas) ni con Panamá (y Centroamérica); por lo contrario, aunque ciertamente no se vincula con las otras tres ciudades de esta zona en conjunto, forma parejas importantes con algunas de ellas, como se verá en seguida. Quito, Lima y La Paz conforman una zona bastante obvia. En orden descedente, las parejas de ciudades relacionadas son las siguientes: 1) Bogotá y Quito (26.3\% de los casos); 2) Lima y La Paz (23.6\%); 3) Qui-

${ }^{69}$ Bombilla (San José, Bogotá); foco (México, San Salvador, Tegucigalpa, Quito, Lima, La Paz, Asunción); bajía (Managua); ampolleta (Santiago); bombita (Montevideo, Buenos Aires)...

70 Almacén (Santiago, Montevideo, Buenos Aires), tienda de abarrotes (México, Quito, La Paz); supermercado (Guatemala, Tegucigalpa, Panamá, Lima, Asunción); tienda (San Salvador, La Paz); miscelánea (México, Managua); pulpería (San José); cigarrería (Bogotá); abasto (Caracas); fiambrería (Santiago).

${ }^{71}$ Volante (en muchas capitales); timón (Guatemala, San Salvador, Tegucigalpa, Panamá, Bogotá, Lima); dirección (Montevideo); manubrio (Santiago); rueda, manivela (San José).

${ }^{72}$ Véase n. 40.

${ }^{73}$ Traje (la designación más frecuente); vestido (Panamá); paltó (Caracas); terno (Quito, Lima, La Paz, Santiago); ambo (Buenos Aires). 
to y La Paz (15.7\%); 4) Quito y Lima (14.4\%); 5) Bogotá y I ma $(10.5 \%) ; 6)$ Bogotá y La Paz $(9.2 \%)$.

En esta zona dialectal hay realmente un solo grupo de tr países verdaderamente importante; las demás asociaciones dan en pocos casos: 1) Quito, Lima y La Paz (68.1\% de los c sos); 2) Bogotá, Lima y La Paz (13.6\%); 3) Bogotá, Quito y I ma, por una parte; y Bogotá, Quito y La Paz, por otra (cada u con $9 \%$ ). Son muy escasos los conceptos para los cuales se obt vo la misma respuesta en las cuatro capitales de la zona ${ }^{74}$.

Anoto en seguida ejemplos de cuestiones en que la respues obtenida parece exclusiva de algunas ciudades de esta área. Ll GAR PARA TOMAR BEBIDAS DE ÍNFIMA CALIDAD: $c$ chería (Quito, La Paz) ${ }^{75}$; PERMISO PARA GUIAR (docume to que autoriza a manejar): brevete o breveta (Quito, Lima) LAS CALIFICACIONES (documento que recibe el estudiar con sus notas): libreta (Quito, La Paz) ${ }^{77}$; EL PELO DE LC NEGROS: $z a m b o$ (Quito, Lima) ${ }^{78}$; CAZADORA SENCILL. chompa (Bogotá, Quito) ${ }^{79}$; SUJETACORBATA: prendedor ( corbata) (Quito, Lima) 80 ; INDIVIDUO PEQUEÑO: chato (I ma, La Paz) ${ }^{81}$; HUEVOS PASADOS POR AGUA: pasac (Lima, La Paz) ${ }^{82}$; LIQUIDACIÓN (venta especial que se ha en ciertas ocasiones, con precios muy rebajados): realización (B gotá, Quito, Lima) ${ }^{83}$; MARCHA ATRÁS: retro (Quito, ]

${ }^{74}$ Parece ser que son sólo dos casos: BARMAN (El que atiende la ver de bebidas alcohólicas en un restaurante): barman (Bogotá, Quito, Lima y Paz), respuesta que también se documentó en varias otras capitales; PORT FOLIOS (artefacto pequeño y estrecho, que se usa sobre todo para llevar critos): portafolios (en esas cuatro ciudades y, también, en varias otras).

${ }^{75}$ Véase n. 54

${ }^{76}$ Licencia (en muchas ciudades); camet (Santiago); libreta (Montevidı Buenos Aires); registro (Asunción, Buenos Aires).

${ }^{77}$ Calificaciones (México, La Habana, Quito); libreta, tarjeta de notas (n chas ciudades); boletín (Managua)...

${ }^{78}$ Véase n. 20.

${ }^{79}$ Chamarra (México, Managua, La Paz); chumpa (Guatemala, San S vador, Tegucigalpa); chaqueta (Panamá, Santo Domingo, Caracas, Ast ción), jacket (San José, La Habana, San Juan); abrigo (La Habana, Panam casaca, parca (Santiago); campera (Montevideo, Buenos Aires).

80 Véase n. 57.

81 Véase n. 37

82 Pasados por agua (en muchas capitales); tibios (México, Guatemala, S Salvador, Tegucigalpa, Managua, Bogotá, Caracas); aguados (Panamá); $h$ vos a la copa (Santiago).

${ }^{83}$ Liquidación (San Salvador, Tegucigalpa, San José, San Juan, Sant 
Paz) ${ }^{84}$; BOLÍGRAFO: esforógrafo o esferográfica (Bogotá, Quito) ${ }^{85}$; PORTAMONEDAS: chauchera (Quito, La Paz) ${ }^{86}$.

\section{Santiago, Asunción, Montevideo, Buenos Aires}

Probablemente sea ésta la zona dialectal más evidente entre las que se postulan en este estudio. Ello se debe a varios factores: la cercanía geográfica de dos de las capitales que conforman el grupo (Montevideo y Buenos Aires), misma que explica sus notables afinidades de vocabulario; la imponente cordillera de los Andes no es obstáculo para que Santiago tenga vínculos culturales (e idiomáticos) más fuertes con Buenos Aires y con Montevideo que con las otras capitales de la región central y norte de Sudamérica (La Paz, Quito); finalmente, aunque la distancia entre Asunción y Buenos Aires no es mucho menor que la que media entre la capital paraguaya y La Paz, es obvio que se relaciona más estrechamente con la metrópoli argentina que con la capital boliviana. Ciertamente la ciudad de Santiago parece establecer importantes vínculos con Quito (y La Paz). Sin embargo, cuando se forman grupos de más de dos ciudades, no cabe duda alguna de que la capital chilena se relaciona más evidentemente con Buenos Aires y Montevideo (y Asunción) que con La Paz y Quito (y Lima). A ello obedece que quede incluida en esta zona dialectal.

Una prueba de que en efecto se trata de una zona dialectal, de conformidad con los criterios aquí manejados, es el hecho de que son numerosos los conceptos que obtuvieron la misma respuesta en las cuatro ciudades, lo que no sucede sino en mucho menor proporción en las zonas dialectales anteriormente explicadas. En algunos casos, los menos, se trata, además, de respuestas exclusivas de estas ciudades, en cuanto que los vocablos no se documentaron en las encuestas de otras capitales; en otros, la res-

go, Montevideo, Buenos Aires); barata (México); aferta (Guatemala, San Salvador, La Habana); baratillo (San José, Santo Domingo); indemnización (Montevideo).

${ }^{84}$ Atrás (La Habana, Santiago, Asunción, Montevideo, Buenos Aires); reversa (México, Panamá, Lima); retroceso (Guatemala, San Salvador, Tegucigalpa, Managua, Caracas); riversa (Santo Domingo).

${ }^{85}$ Bolígrafo (Santo Domingo, Asunción); pluma atómica (México); lapicero (Guatemala, Managua, La Habana); pluma (Panamá, Caracas); lápiz tinta (Tegucigalpa); lápiz de pasta (Santiago); lapicera (Montevideo); birome (Asunción, Buenos Aires).

${ }^{86}$ Monedero (en muchas capitales); portamonedas (México); pistera (San Salvador); cartera (San Juan). 
puesta es compartida por sujetos de otras partes. Véanse algunc ejemplos de estos conceptos y sus respuestas, uniformes en los ir formantes de las cuatro capitales de esta zona del extremo sur de continente: LA CARPETA, FOLDERS (tapas de cartón o cartu lina en que se guardan documentos): carpeta $^{87}$; EL EMBRA GUE, CLUTH (del automóvil): embrague ${ }^{88}$; MANICURA: man: cura $^{89}$; LA MANTA, FRAZADA, COBIJA (prenda que se pon inmediatamente sobre las sábanas): frazada ${ }^{90}$; COGEDOR ( BADIL: pala o palita ${ }^{91}$; ADEHALA, ÑAP, PILÓN (lo que ، que vende regala al que le ha comprado algo): yapa ${ }^{92}$; EL LIN. PIABOTAS: lustrabotas $^{93}$; EL CLAXON O BOCINA (del autc móvil): bocina ${ }^{94}$; LA HORA Y TREINTA Y CINCO: menos trein ta y cinco $^{95}$; TORONJA (fruta similar a la naranja pero un poc más amarga): pomelo $^{96}$; LA FALDA: pollera $^{97}$; ACERA: vered $a^{9}$

87 Vocablo también documentado en Bogotá y Caracas. La otra respue ta, muy extendida en América, es fólder.

88 Véase la n. 62.

${ }^{89}$ En el resto de América se prefiere manicurista. Otras respuestas: manicu (pronunciado maniquiur) en San José, Panamá, La Habana, Caracas.

${ }^{90}$ Vocablo que se documentó en otras ciudades (Guatemala, San Salv: dor, Lima). Algunas otras respuestas: cobija (México, Tegucigalpa, San Jos Bogotá, Quito), sábana (gruesa) (Panamá, Caracas), cobertor (México), frisa (Sa1 to Domingo, San Juan).

${ }^{91} \mathrm{La}$ misma respuesta se obtuvo en: San Salvador, Tegucigalpa, Man. gua, San José, Santo Domingo, Caracas, Quito. Otras respuestas: recoged (México, Guatemala, Panamá, La Habana, San Juan, Bogotá, Lima); basurt (La Paz).

${ }^{92} \mathrm{La}$ misma respuesta se dio en Quito, Lima y La Paz. Otras respuesta pitón (México), ganancia (Guatemala, San Salvador), chascada (Tegucigalpa vendaje (Managua), ñapa (Panamá, Santo Domingo, San Juan, Caracas), camb yada (La Habana), encime (Bogotá).

${ }_{93}$ Véase n. 26.

${ }^{94}$ Esta palabra fue también registrada en Guatemala, Santo Doming San Juan y La Paz. Otras respuestas: claxon (México, La Habana, San Jua: Lima); pito (San Salvador, Tegucigalpa, Managua, San José, Bogotá, Quitc corneta (Caracas).

${ }^{95}$ Respuesta que también se da en Guatemala y San Juan. Veinticinco pa se refiere en México, Caracas, Lima (puede oírse en otras varias capitales); hora y treinta y cinco fue respuesta en San Salvador, Managua, La Habana y B gotá (aunque también registrada, como segunda opción en otras capitales).

96 Toronja se emplea en todas partes. También se documentó el anglicisn grape fruit en Managua, Panamá, Santo Domingo y La Paz.

${ }_{97}$ Se prefiere en casi todas partes falda; se registró (e)nagua(s) en Manag! y San José, saya en La Habana.

${ }^{98}$ Designación también recogida en Quito, Lima y La Paz. En la may 
MARCAR UN NÚMERO TELEFÓNICO: discar ${ }^{99}$; LA TAQUILLA (lugar donde se paga el importe del billete de entrada al cine): boletería ${ }^{100}$; ESTILOGRÁFICA, PLUMA FUENTE: lapicera (fuente) $)^{101}$.

Ahora bien, si se agrupan por parejas las ciudades de la zona, en los casos en que sólo en esas dos capitales (y no en tres ni en cuatro) se dio una misma respuesta, pueden formarse cinco grupos que, ordenados descendentemente del más importante al menos destacable son los siguientes (aparece anotado entre paréntesis el porcentaje que cada uno obtuvo): 1) Montevideo y Buenos Aires (32.7\% de los casos); 2) Asunción y Buenos Aires (23.6\%); 3) Santiago y Buenos Aires (14.5\%); 4) Santiago y Montevideo (9\%); 5) Santiago y Asunción (5.4\%). Obsérvese por una parte el obvio predominio de la pareja formada por Montevideo y Buenos Aires; y, por otra, la importancia y la influencia que en las demás capitales tiene la ciudad de Buenos Aires, que interviene en las dos más importantes parejas.

Cuando son tres las ciudades que se agrupan (es decir cuando en tres de las cuatro capitales se obtiene la misma respuesta) hay una terna que sobresale de manera obvia: 1) Asunción, Montevideo y Buenos Aires, que obtiene el $68.4 \%$ del total de estos casos. Los demás grupos, en orden descedente, son los que siguen: 2) Santiago, Montevideo y Buenos Aires (24.5\%); 3) Santiago, Asunción y Buenos Aires (14\%); 4) Santiago, Asunción y Montevideo $(10.5 \%)$. Nuevamente queda clara la importancia que para el establecimiento de este dialecto sudamericano, basado exclusivamente en rasgos de vocabulario estándar, tiene el habla de la capital argentina, que interviene en los tres grupos más importantes.

Termino este apartado, como los anteriores, dando algunos ejemplos de vocablos que obtuvieron, en algunas de las ciudades que conforman la zona, respuestas que pudieran considerarse exclusivas, en cuanto que no fueron registradas en las encuestas de

parte de las ciudades se emplea la voz acera. En México (y algo en Guatemala) se dice banqueta.

${ }^{99}$ Verbo también empleado en Lima y La Paz. En casi todas partes predomina marcar.

${ }^{100}$ Vocablo empleado también en La Paz y, en menor medida, en San José y Quito. Taquilla es la designación más común. Un sujeto de Managua contestó ticketería.

${ }^{101}$ Pluma (fuente) es el vocablo más común. Otras designaciones: estilográfica (Bogotá), estilógrafo (Quito). 
las demás capitales americanas ${ }^{102}$. FIAMBRES (carnes que se sirven frías después de guisadas): fiambres (Montevideo, Buenos Aires) ${ }^{103}$; ORFANATO: asilo de huérfanos o niños (Montevideo, Buenos Aires) ${ }^{104}$; PORTALÁMPARAS, SOCKET: portalámparas (Santiago, Asunción, Montevideo, Buenos Aires) ${ }^{105}$; LA PLACA CON EL NÚMERO (en el automóvil): chapa (Asunción, Montevideo y Buenos Aires) ${ }^{106}$; PERMISO PARA GUIAR: libreta (Montevideo, Buenos Aires), registro (Asunción, Buenos Aires) ${ }^{107}$; ¿CÓMO SE GONTESTA EL TELÉFONO?: hola (Asunción, Montevideo, Buenos Aires) ${ }^{108}$; LAS SIETE DE LA TARDE (O DE LA NOCHE): de la tarde (Santiago, Asunción. Montevideo y Buenos Aires) ${ }^{109}$; TAPAS (cosas que se sirven er un aperitivo, fuera de los licores): picada, picadillo (Montevideo Buenos Aires) ${ }^{110}$; HABICHUELAS, JUDÍAS VERDES chauchas (Asunción, Montevideo, Buenos Aires)111; EL TRESI. LLO: (juego de) living (Santiago, Asunción, Montevideo, Bueno: Aires) ${ }^{112}$; EL FRIGORÍfICO: heladera (Asunción, Montevideo Buenos Aires) ${ }^{113}$; FALTAR A CLASES VOLUNTARIAMEN

102 Lo que de ninguna manera quiere decir que se desconozcan totalmen $t e$, pues, como se ha venido diciendo, los resultados de estas encuestas, debide al bajo número de informantes, tienen un sentido enteramente provisional No dejan sin embargo de tener su interés, pues permiten conocer, a mi ver el empleo, si no exclusivo, sí predominante en cada ciudad.

${ }^{103}$ En la mayor parte de las capitales hispanoamericanas el término pre ferido es carnes frías.

104 En América predomina orfanatorio.

105 En el resto del continente parece predominar sócket. En Santo Domin go se registró zócalo, y boquilla en Quito.

${ }^{106}$ En la mayoría de las capitales se prefiere placa. En San Juan se docu mentó tablilla.

107 Véase n. 76.

${ }^{108}$ En el resto de las capitales americanas se prefiere aló; en México s dice bueno.

${ }^{109}$ De la noche es lo más usual en las demás capitales americanas.

110 Véase la n. 51.

111 Otras respuestas: ejotes (México, Guatemala, San Salvador), friji verde (Tegucigalpa, Managua), vainicas o vainitas (San José, Santo Dommgc Caracas, Quito, Lima), habichuelas (Tegucigalpa, Panamá, La Habana, Sa Juan, Bogotá), porotos (verdes) (La Paz).

112 Otras respuestas: terno (de sala) (México), (juego de) sala (Guatemale San Salvador, Tegucigalpa, Managua, Panamá, La Habana, Bogotá, Qu to), mesa (-ita) (San Juan, La Paz).

${ }^{113}$ Las más destacables voces americanas son: refrigerador (México, Gú temala, San Salvador, Tegucigalpa, Managua, Panamá, La Habana, Quits Lima, La Paz), nevera (San José, Santo Domingo, San Juan, Bogotá, Caracas 
TE (acción premeditada de un alumno de no asistir a clases): $h a-$ cerse la rabona (Asunción, Montevideo, Buenos Aires) ${ }^{114}$; IMPERMEABLE: pilot o piloto (Asunción, Montevideo, Buenos Aires) ${ }^{115}$; ARETES: aros (Santiago, Asunción, Montevideo, Buenos Aires) $)^{116}$.

\section{RESUMEN Y CONCLUSIONES}

El español americano suele ser caracterizado, y no sin razón, como un diasistema cuyos sistemas fonológicos y gramaticales son muy semejantes. Las diferencias internas de este diasistema deben buscarse, sobre todo, en la fonética y en el léxico. Aun éstas, indudables, deben sin embargo ser entendidas como diferencias menores, en cuanto que jamás impiden la intercomunicación de los hablantes. En lo que concierne específicamente a las diferencias de vocabulario, que obviamente están presentes, no son tampoco de naturaleza tal que supongan para el español americano un grave riesgo de fragmentación. Hay en todos los idiolectos un importante acervo de vocabulario común, sobre todo en los dialectos geográficos que suelen conocerse como urbanos y en los dialectos sociales que llamamos cultos. Es sin duda más fácil encontrar diferencias de vocabulario en idiolectos pertenecientes a dialectos rurales y populares.

Sin embargo también hay diferencias en el léxico urbano culto. Se trata de un asunto poco estudiado, si se considera que la dialectología se encarga, normalmente, de hablas rurales. Los datos que se proporcionaron en este estudio se obtuvieron por medio de encuestas que cabrían en el apartado de la dialectología urbana, no sólo porque los sujetos son oriundos de las grandes ciudades americanas, las capitales, sino también por el tipo de pre-

${ }^{114}$ Irse de pinta o pintar venado (México), irse de capiura, capear(se) (San Salvador, Tegucigalpa), libretearse (Managua), pavear (Panamá), tiguerear (Santo Domingo), comer jobos (San Juan), jubilarse (San Juan), echar o hacerse la pera (Quito), vaquear o hacer la vaca (Lima), chacharse (La Paz).

115 Gabardina (México, Caracas, Quito); impermeable (México, Bogotá, Lima, La Paz, San Juan, Caracas, Quito); capa (Guatemala, San Salvador, San José); capote (Tegucigalpa, Managua, Panamá, Santo Domingo); chubasquero (La Habana); capa de agua (San Juan).

${ }_{116}$ Aretes (México, Guatemala, Panamá, La Habana, Bogotá, Quito, Lima, La Paz); aritos (San Salvador, Tegucigalpa, Santo Domingo); chapas (Managua); argollas (San José, Panamá); pantallas (San Juan); zares (Bogotá); zarcillos (Caracas). 
guntas que conforman el cuestionario, referentes todas ellas a vida urbana cotidiana. Los resultados muestran que hay diferes cias en el vocabulario básico que emplean los hablantes de las c versas ciudades. No todas las designaciones registradas en las e: cuestas están incorporadas en los diccionarios, ni generales regionales. Ello muestra también que no hay todavía una trac ción en lo que respecta al estudio de este tipo de léxico.

Entre los diversos criterios que pueden adoptarse para el tr zo de isoglosas, está el que analiza diferencias de vocabulari Traté aquí de dividir en zonas el enorme territorio americar mediante la observación de las semejanzas y diferencias de voc bulario existentes entre los dialectos hablados en las ciudades c pitales. Si el resultado no puede concebirse, estrictamente, con una división dialectal, al menos se podrá aceptar que hay vari formas de vincular el léxico de los diversos dialectos urbanos c español de América, es decir que las hablas de cada una de l ciudades tienen semejanzas (y diferencias) más acusadas con : gunas de las hablas de las otras capitales (y no con todas). El o jeto del estudio era establecer con cierta precisión ese tipo de vi culaciones.

Resulta evidente que hay parejas de ciudades cuyos vor bularlos parecen particularmente semejantes. Según mis resul 1 dos ése sería el caso de parejas como las siguientes: Guatema y San Salvador, San Salvador y Tegucigalpa, Santo Dominge San Juan, La Habana y Santo Domingo, Bogotá y Quito, Lir y La Paz, Montevideo y Buenos Aires, entre otras. Tambi pueden formarse ternas de capitales con notables semejanzas léxico urbano dialectal, por ejemplo: Guatemala, San Salvac y Tegucigalpa; La Habana, Santo Domingo y San Juan; Qui Lima y La Paz; Asunción, Montevideo y Buenos Aires.

Naturalmente que las agrupaciones de mayor número de $\mathrm{d}$ lectos urbanos, vinculados por semejanzas léxicas dialectal van resultando cada vez menos convincentes. Sin embargo exist Ello permite formular la existencia de ciertas zonas dialectales. otras palabras, si se buscaran grupos de dialectos urbanos con: tuidos por seis capitales vinculadas por semejanzas léxicas, muy probable que no hubiera otro grupo más claro que Centr mérica. Lo mismo podría decirse de los grupos que podrían $\mathrm{s}$ gir de cuatro capitales: seguramente éstos serían Bogotá, Qui Lima y La Paz, por una parte; y Santiago, Asunción, Monte deo y Buenos Aires, por otra.

Evidentemente hay capitales cuyos dialectos no caben, cór 
damente, en ninguna de estas zonas. Tal vez el mejor ejemplo sea la ciudad de México: ¿forma un grupo con las capitales centroamericanas? ¿constituye por sí sola un dialecto? Aunque hay razones para inclinarse por una u otra hipótesis, los resultados obtenidos me llevan a la primera propuesta: el léxico de la ciudad de México o, si se quiere, del Valle de México, con sus casi veinte millones de habitantes, constituye una zona dialectal completa, tanto porque cuenta con un buen número de vocablos privativos cuanto porque no son muy satisfactorias las vinculaciones que establece con los léxicos de las capitales centroamericanas (y antillanas).

Los otros casos frontera son relativamente más fáciles de resolver. Uno sería la ciudad de Caracas, la cual, siempre según mis materiales léxicos, parece más cercana a la zona antillana que a la primera de las zonas sudamericanas. A ello obedece que se formule la existencia de una zona antillana, formada por las tres capitales insulares más Caracas. El otro es la ciudad de Santiago: podría vincularse con La Paz o Lima, hacia el norte, o con Buenos Aires (Asunción y Montevideo), hacia el oriente. No sin muchas dudas y titubeos, me incliné por lo segundo.

Seguramente, si se maneja otro vocabulario, o si se aplica otro método en la encuesta misma o en el análisis de los resultados, habrá necesidad de proponer otro tipo de vinculaciones y, por tanto, otras zonas dialectales. Quede la presente hipótesis como una simple propuesta más, que se suma a las varias ya existentes, para dividir en zonas dialectales el territorio americano. Sigo creyendo, por otra parte, que cualquier intento de división, si está fincado sobre bases firmes, sobre datos lingǘsticos confiables, contribuye siempre en alguna medida al mejor conocimiento del español americano.

José G. Moreno de Alba Universidad Nacional Autónoma de México 
